\title{
Comparison of treatment records and inventory of empty drug containers to quantify antimicrobial usage in dairy herds
}

\author{
Diego B. Nobrega, ${ }^{*}{ }^{1}$ Jeroen De Buck, ${ }^{*} \dagger$ S. Ali Naqvi, ${ }^{*} \dagger$ Gang Liu, ${ }^{*} \dagger \ddagger$ Sohail Naushad, ${ }^{*} \dagger$ Vineet Saini, ${ }^{*} \S$ \\ and Herman W. Barkema*†\# \\ *Department of Production Animal Health, Faculty of Veterinary Medicine, University of Calgary, Calgary, AB, T2N 1N4, Canada \\ †Canadian Bovine Mastitis and Milk Quality Research Network, St-Hyacinthe, QC, J2S 7C6, Canada \\ łDepartment of Clinical Veterinary Medicine, College of Veterinary Medicine, China Agricultural University, Beijing 100193, P.R. China \\ §Department of Research and Innovation, Population, Public and Indigenous Health, Alberta Health Services, Calgary, T2W 3N2, Canada \\ \#Department of Reproduction, Obstetrics and Herd Health, Faculty of Veterinary Medicine, Ghent University, Salisburylaan 133,9820 Merelbeke, \\ Belgium
}

\begin{abstract}
Assessment of antimicrobial use (AMU) is vital for interpreting the origin of changes in antimicrobial resistance (AMR). The objectives of the present study were to estimate the association between AMU determined using on-farm treatment records (TR) and inventory of empty drug containers (INV). Herds were selected to represent Canadian dairy farms. Producers were asked to record animal health events and treatments on a standard General Health Event form. For inventory data, 40-L receptacles were placed at various locations considered convenient to deposit all empty drug containers. Antimicrobial defined-daily dosages (ADD) were calculated for 51 Canadian herds using the 2 methods. Estimation of AMU was 31,840 ADD using the INV and 14,487 ADD using the TR, indicating that for every TR entry, 2.20 times more treatments were observed using the INV. Mastitis, reproductive conditions, and dry cow therapy were the most frequent reasons for antimicrobial therapy when assessing TR. For all antimicrobials evaluated, mean ADD was higher using the INV versus TR. Regardless, a strong positive correlation (0.80) was observed between the 2 methods, indicating that herds with increased number of ADD recorded using the INV also had increased number of ADD recorded using TR. Furthermore, a positive association was observed for the 6 most commonly used antimicrobials. In comparison to methods used in surveillance programs on AMU in livestock that assume a constant use in all herds (i.e., sales data), INV provided a herd-level specific quantity of AMU positively correlated with AMU recorded at the animal level in general. The INV was easy to implement and provided a
\end{abstract}

Received May 4, 2017.

Accepted August 18, 2017.

${ }^{1}$ Corresponding author: diego.nobrega@ucalgary.ca measure of total AMU in the herd. Availability of such information would be valuable for interpreting changes in AMR at the herd level and enabling evaluation of interventions for decreasing AMR.

Key words: antimicrobial daily dose, antimicrobial use, antimicrobial resistance, inventory of empty drug containers, treatment record

\section{INTRODUCTION}

Antimicrobials are vital to maintain animal productivity, improve animal welfare by limiting disease impact, and improve food safety. However, they are also deemed to increase antimicrobial resistance (AMR). Assessing antimicrobial use (AMU) is essential for interpretation of AMR patterns (Landers et al., 2012). Quantification of AMU serves as a basis of decision making for control measures and enables evaluation of interventions in AMU. However, obtaining good-quality AMU data in production animals is challenging (Queenan et al., 2016). National data on sales or prescriptions of antimicrobials for production animals yield average results over all farms and animal species, even if a particular antimicrobial is not being used on a subsection of farms (Bondt et al., 2013). However, availability of on-farm recorded data would aid surveillance programs and epidemiological studies, thereby justifying the investment in obtaining such data (Queenan et al., 2016).

On-farm treatment records (TR) are important for determining AMU and treatment efficacy in infectious diseases (Gonzalez et al., 2010). Treatment records are often used to identify management deficits that lead to increased number of treatments in a short interval (Rhoda and Pantoja, 2012). In theory, TR should be the definitive method of recording AMU. Recording all antimicrobial treatments would enable dairy producers to keep track of AMU in dairy herds. However, maintaining accurate TR requires considerable effort and 
commitment from all people working with the cattle. Therefore, failure to establish this method on a dairy farm as a result of incomplete and unverifiable records is not rare (Rhoda and Pantoja, 2012).

Other methods for measuring AMU in dairy farms include questionnaires and surveys (Pol and Ruegg, 2007; Eagar et al., 2012; Bryan and Hea, 2017) and inventory (INV) of empty drug containers (Saini et al., 2012). Usually, data on AMU in national surveillance systems are based on sales or prescriptions (or both) of antimicrobials. That does not, however, necessarily mean that these drugs were actually used. The INV of empty drug containers using receptacles located at strategic places on a farm is a powerful tool to quantify total AMU (Carson et al., 2008). In theory, the INV has higher sensitivity to detect actual treatments.

In any successful AMR control program, a combination of organization, effort, and willingness is required. The objectives of this study were therefore to (1) compare AMU estimated using TR and INV, and (2) determine whether an association is present between AMU recorded using TR and INV.

\section{MATERIALS AND METHODS}

\section{Herd Enrollment}

Data for this study were obtained from the National Cohort of Dairy farms of the Canadian Bovine Mastitis Research Network (Reyher et al., 2011). Briefly, 89 dairy herds from several regions in Canada (Alberta, Ontario, Quebec, and the Maritimes provinces Prince Edward Island, New Brunswick, and Nova Scotia) were followed for $2 \mathrm{yr}$. Herds were specifically selected to represent Canadian milk-recorded herds. Therefore, herds were selected to be a regional representation of the proportion of housing systems (free-stall vs. tie-stall) in the region, and 3 bulk tank SCC (BTSCC) strata based on 12-mo geometric mean BTSCC, classified as low, intermediate, and high $(<150,000,150,000$ to 300,000 , and $>300,000$ cells $/ \mathrm{mL}$, respectively). Additionally, each herd had at least $80 \%$ lactating Holstein-Friesian cows milked twice a day and participated in a DHI recording system.

To be included in the present study, a herd had to record at least 1 antimicrobial treatment rendered at animal level, and place at least 1 empty drug container in the receptacles for the duration of the study. In addition, a herd could have no more than $10 \%$ of the records with missing data for each of the general health event form (GHEF) minimum required fields (cow ID, reason for treatment, treatment date, treatment product, and treatment duration; Figure 1). As a result, 51 herds were included in the study (Table 1).

\section{Antimicrobial Use Data}

All farm personnel were instructed by Canadian Bovine Mastitis Research Network technicians to record animal-level health events and treatments, particularly

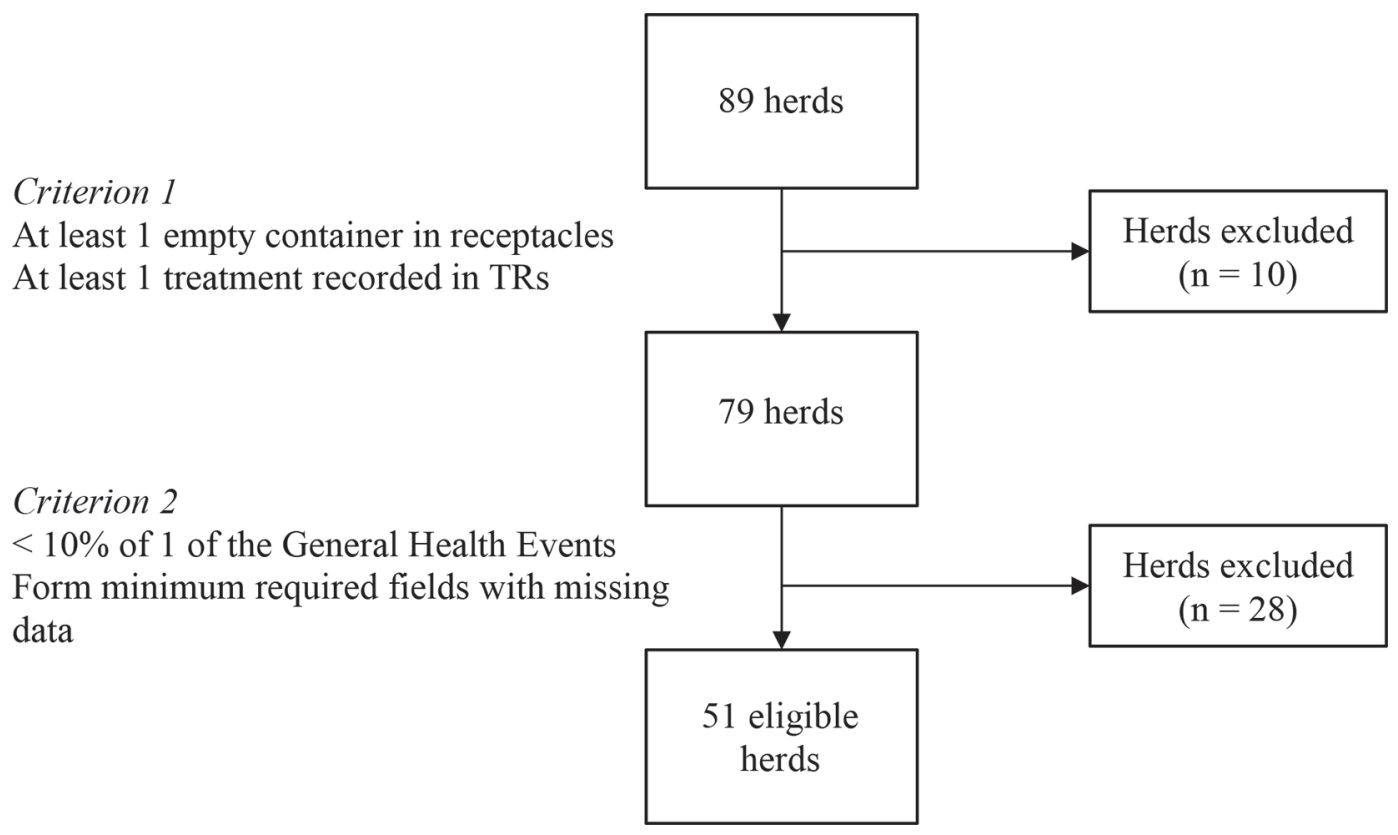

Figure 1. Flow diagram of herd selection process. 
those resulting in AMU, for approximately 2 yr using a standard GHEF containing the following data elements: herd ID, cow ID, reason for treatment, treatment date, treatment product, and treatment duration.

The methodology for collecting and counting the empty drug containers and recording AMU at farm level has been described (Saini et al., 2012). Briefly, $40-\mathrm{L}$ receptacles were placed in locations on the farms where treatments were rendered or drug containers were stored so that it was convenient for farm personnel to deposit empty drug containers. Farm personnel were instructed to dispose of all empty drug containers in these receptacles. Technicians and students visited all farms at least once per month. The number of empty drug containers was counted, and information regarding herd, antimicrobial used, product name and weight or volume, and start and end date of the current collection period was recorded on drug tally sheets.

\section{Estimation of Antimicrobial Defined-Daily Dosages}

Data were entered in spreadsheets (Microsoft Excel, Microsoft Corp., Redmond, WA). All entries were manually checked. Duplicates were excluded and no unlikely values were detected. Using the INV, the expected animal defined-daily dose (ADD; g/d) per empty drug container was calculated, based on the active pharmaceutical ingredient considering the onlabel recommended daily dose. Doses were based on the Canadian Compendium of Veterinary Products (CVP, 2015). Because some drugs could be used almost exclusively for young stock, a preliminary evaluation of the TR was done to identify the animal category that was most frequently submitted to treatment. If $95 \%$ or more of the drug-specific records indicated that the treated animal was $<1 \mathrm{yr}$, ADD were calculated with a standard BW of $200 \mathrm{~kg}$. In this case, an important assumption was that nonrecorded treatments with the same antimicrobial were used almost exclusively in the same animal category as indicated by the TR. Otherwise, ADD were calculated considering the on-label recommended daily dose for a standard adult dairy cow $(\mathrm{BW}=600 \mathrm{~kg}$; Jensen et al., 2004).

Antimicrobial defined-daily dosages were summarized for the following antimicrobial drug classes: aminoglycosides, first-generation cephalosporins, thirdgeneration cephalosporins, fluoroquinolones, lincosamides, macrolides, penicillins, penicillin combinations (penicillin G, novobiocin, dihydrostreptomycin and polymyxin $\mathrm{B}$, and benzylpenicillin procaine and benzylpenicillin benzathine), phenicols, tetracyclines, and trimethoprim-sulfonamide combinations.

Using the TR, every daily antimicrobial treatment recorded was considered an ADD (e.g., 3 animals under treatment for 3 consecutive days were considered as 9 ADD). Because information about dosage rendered was poorly recorded (i.e., missing/incomplete in $>90 \%$ of the records), all treatments were assumed to be rendered at the recommended daily dosage for that particular drug/disease combination. In 11\% of recorded therapies in the TR, data for days under treatment were missing. An imputation method was used in those instances, where the days under treatment were imputed as the mode of the days under treatment using the same drug for that same condition in the same herd. Categories of treatment events were created, which included treatments for displaced abomasum, dry cow intramammary treatments, fever, foot diseases, mastitis, reproductive conditions (retained fetal membranes, dystocia, fresh cow support, and metritis), respiratory diseases, others (abscess, bladder infection, diarrhea, general sickness, reticuloperitonitis, general pain and injuries, indigestion, ketosis, navel infections, low milk production without specified reason, animal off-feed, nonspecified infections, peritonitis, and postsurgery treatments), unclear, and unknown. The ADD were also summarized according to the drug classes described previously. Finally, for all analyses, the ADD recorded for individual

Table 1. Description of the 51 participating Canadian dairy herds (according to region)

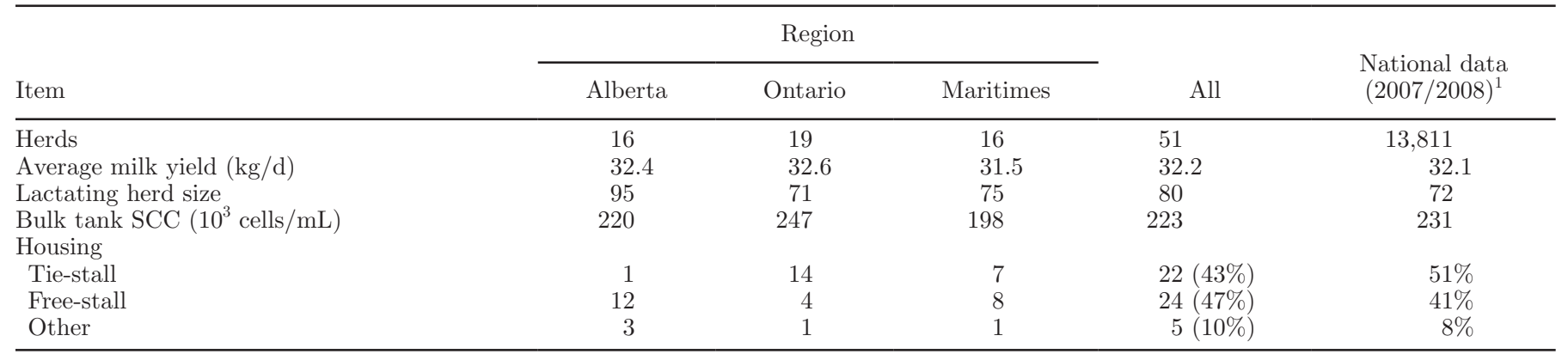

${ }^{1}$ Number of herds, milk production, herd size, bulk tank SCC values obtained from Canadian Dairy Information Centre (CDIC, 2017). Housing types obtained from the study by Olde Riekerink et al. (2010). 
animals using TR were summarized to obtain herd-level ADD, which was an estimate of herd-level AMU.

The ADD per day or ADD rate (ADDR) was calculated at the herd level by dividing herd-level ADD by the number of days for which the herd was enrolled in the study. For the purpose of standardization, ADDR was converted to ADD per 365 d. A second parameter, the antimicrobial drug use rate (ADUR; ADD/100 cow-years) was estimated using the average number of adult cows in the herd during the duration of the study. The ADUR is a standardized measure of antimicrobials consumption in a dairy herd, with advantages of being time sensitive and not affected by the total number of animals when comparing AMU among herds. For both ADDR and ADUR, the number of days or animal-days at risk originated only from herds where a particular antimicrobial was used at least once, providing a measure of the dependency upon an antimicrobial conditional on its use.

\section{Statistical Analyses}

Differences in $A D D$. Antimicrobial defined-daily dosages of each antimicrobial class were log-transformed (logADD) to reduce the ADD right skewness and to approximate conditional normality. Expected $\log \mathrm{ADD}$ recorded using TR and the INV method were compared using linear mixed models in R (lme4 package; Crawley, 2013; Bates et al., 2015) for several antimicrobial classes in individual models (penicillins, penicillin combination, third-generation cephalosporins, first-generation cephalosporins, trimethoprim-sulfonamide combinations, tetracyclines, lincosamides, phenicols, and macrolides). Two approaches dealing with the lack of independence of the observations were tested: (1) regions and herdspecific conditional models (3-level random intercept model) with recording method modeled as fixed effects where $\log$ ADD was considered the outcome; (2) herdspecific conditional models (2-level random intercept model) with recording method modeled as fixed effects and $\log \mathrm{ADD}$ as outcome. The 2 methodologies were tested because (1) unmeasured effects of regions (i.e., technician training) could have introduced a lack of independence in outcome, and (2) 2 measurements (TR and INV) were done for a particular antimicrobial class in each herd. However, because conclusions using both approaches were the same and the estimated variances of the region-specific component for the intercept were not significant, the second (simplest) approach was selected for presentation. To determine overall differences in mean $\log \mathrm{ADD}$ between TR and the INV for each herd (irrespective of drug class), ADD for each drug class were summarized, and the model was refit- ted using the same strategy. Results were presented as geometric means.

To further explore factors related to compliance, herds were stratified according to the ratio between the $\log \mathrm{ADD}$ recorded using both methods (logADD recorded using INV $/ \log \mathrm{ADD}$ recorded using TR; high ratio herds $>1.5$, low ratio herds $\leq 1.5$ ). Province-specific conditional models were used to contrast the 2 categories of herds in terms of BTSCC (log-transformed), herd size, and milk production in separate models.

Association of ADD from TR and INV. Herdlevel ADD was estimated for both TR and INV by adding all drug-specific ADD. Initially, the Pearson product-moment correlation between logADD recorded using the TR and INV were estimated using linear regression in R (Crawley, 2013; Bates et al., 2015). Scatterplots of $\log$ ADD recorded using the TR and INV and hypothesized functional forms between the 2 variables were initially examined, ignoring the lack of independence.

To verify whether a linear, quadratic, or cubic model best fit the data, region-specific random effects models were fitted using lme4 package in R (Bates et al., 2015), where the expected $\log \mathrm{ADD}$ recorded using the INV method was considered the outcome and the $\log \mathrm{ADD}$ recorded using the TR method and its quadratic and cubic terms were predictors. Because previous comparisons were all done at the herd level, we postulated that the method recorded already at the herd level should be considered the outcome in our analysis. Models were compared based on the log-likelihood ratio test. The intraclass correlation coefficient estimating the proportion of the variance that resides in each level was obtained using the same approach. Statistical significance was considered at a $5 \%$ level.

\section{RESULTS}

\section{Descriptive Analyses and Mean ADD}

The 51 study herds were representative of the Canadian dairy herds in terms of milk production, herd size, BTSCC, and housing (Table 1). Most nonparticipating herds used a different method for recording antimicrobial treatments in animals. The remaining 10 herds that did not record at least 1 treatment using either method had an average milk production of $32 \mathrm{~kg} / \mathrm{d}$ and average bulk tank SCC of 275,000 cells $/ \mathrm{mL}$. Six of the 10 herds had on average $\leq 50$ lactating animals for the duration of the study.

Using INV, total AMU recorded in the 51 herds was 31,840 ADD, compared with 14,487 using TR (Table 2 ). The AMU in individual herds ranged from 82 to 3,500 ADD (median $=392$, interquartile range $=482$ 
Table 2. Overall and relative frequency (\%) of antimicrobial defined-daily doses (ADD), herd average antimicrobial daily doses per year (ADDR), and herd average antimicrobial daily doses per 100 cow-years (ADUR) recorded using inventory of empty drug containers (INV) and treatment records (TR) according to the antimicrobial used for treatment

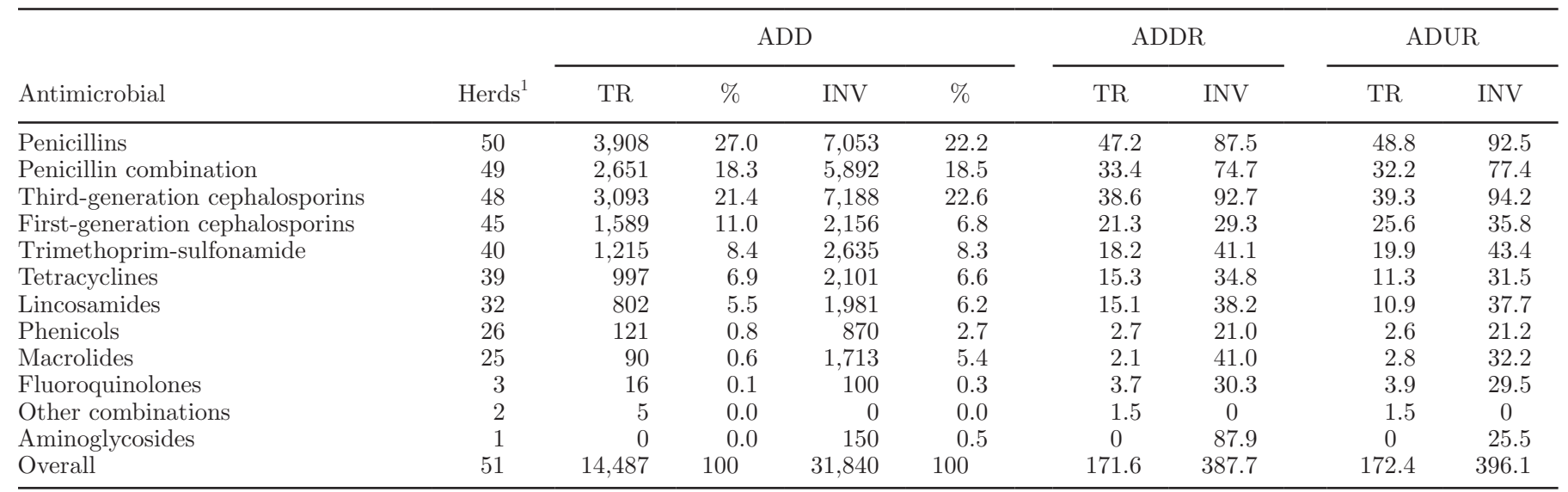

${ }^{1}$ Herds with at least 1 treatment with this antimicrobial in the TR or INV.

ADD) using the INV, and from 5 to 1,640 ADD using the $\mathrm{TR}$ (median $=172$, interquartile range $=276$ ADD). Adjusting for duration of herd participation, estimated ADDR in individual herds ranged from 71 to $2,050 \mathrm{ADD} / 365 \mathrm{~d}$ recorded using the INV (median $252 \mathrm{ADD} / 365 \mathrm{~d})$ and from 4 to $961 \mathrm{ADD} / 365 \mathrm{~d}$ using the TR (median $108 \mathrm{ADD} / 365 \mathrm{~d}$ ). When considering the duration of herd participation and the number of cattle in the herd, herd-level ADUR ranged from 4.3 to 455 ADD per 100 cow-years using TR (median $=156$ ADD/100 cow-years), and from 94.5 to $1480 \mathrm{ADD} / 100$ cow-years $($ median $=344 \mathrm{ADD} / 100$ cow-years) using INV (Table 2).

The ratio between the $\log \mathrm{ADD}$ exceeded 1.5 for 8 herds. These herds were similar to the remaining 43 herds in terms of 2-yr mean lactating herd size $(80.1$ cows for low ratio, 77.9 cows for high ratio; $P=0.88$ ) and average milk yield $(32.5 \mathrm{~kg} / \mathrm{d}$ for low ratio, 30.7 $\mathrm{kg} / \mathrm{d}$ for high ratio; $P=0.07)$. However, the 2 -yr geometric mean BTSCC was higher for high-ratio herds than for low-ratio herds (267,700 vs. 202,300 cells/mL, respectively, $P=0.02$ ).

Mastitis, reproductive conditions, and dry cow therapy were the most frequent reasons for using antimicrobial therapy in studied herds when assessing the TR $(42,14$, and $14 \%$ of 14,487 ADD, respectively). The same 3 reasons were also the most frequent for treatment in a dairy herd when comparing ADD per $365 \mathrm{~d}$ and ADD per 100 cow-years (Table 3). For mastitis, penicillin combinations accounted for 2,623 of 6,111 ADD (43.0\%), followed by first-generation cephalosporins (1,254 ADD; 20.5\%) and lincosamides (790 ADD; $12.9 \%$ ). For dry cow therapy, penicillins and first- generation cephalosporins were most frequently used [1,731 (86.0\%) and $285 \mathrm{ADD}(14.0 \%)$ of $2018 \mathrm{ADD}$, respectively]. Reproductive conditions and foot diseases were commonly treated with third-generation cephalosporins [807 of 2,022 ADD (39.9\%) and 579 of 1,040 ADD (55.7\%), respectively], whereas penicillins were the most commonly used antimicrobial for treatment of displaced abomasum [320 (66.4\%) out of $482 \mathrm{ADD}]$.

According to the TR, penicillin and its combinations, cephalosporins, trimethoprim and sulfonamide combinations, and tetracyclines were the most commonly used antimicrobials in participating herds (Table 2). Penicillins were used, especially for dry cow therapy (44\% of 3,908 ADD), and penicillin combinations were used almost exclusively for treatment of mastitis $(99 \%$ of 2,651 ADD). First-generation cephalosporins were used commonly as mastitis treatment (79\% of 1,589 ADD) and dry cow therapy (18\% of 1,589 ADD). Reproductive conditions and foot diseases were the most frequent reasons for treatment with third-generation cephalosporins (26 and 19\% of 3,093 ADD, respectively). Mastitis and reproductive conditions were the most common reasons for treatment with a combination of trimethoprim and sulfonamides (46 and $8 \%$ of 1,215 ADD, respectively). Additionally, 48 and $14 \%$ of 997 ADD with tetracyclines were used for reproductive conditions and foot diseases, respectively. As for lincosamides and macrolides, their use was associated with mastitis treatments ( $98 \%$ of 802 lincosamides ADD) and respiratory diseases (40\% of 90 macrolides ADD).

For all antimicrobials evaluated, geometric mean ADD was higher using the INV than using the TR (Table 4). 
Table 3. Overall (N) and relative frequency (\%) of antimicrobial defined-daily doses (ADD), herd-average antimicrobial daily doses per year (ADDR), and herd-average antimicrobial daily doses per 100 cow-years (ADUR) recorded using treatment records (TR) according to the reason for treatment

\begin{tabular}{|c|c|c|c|c|c|}
\hline \multirow[b]{2}{*}{ Treatment reason } & \multirow[b]{2}{*}{ Herds $^{1}$} & \multicolumn{2}{|c|}{$\mathrm{ADD}$} & \multirow[b]{2}{*}{ ADDR } & \multirow[b]{2}{*}{ ADUR } \\
\hline & & $\mathrm{N}$ & $\%$ & & \\
\hline Mastitis & 50 & 6,111 & 42.2 & 74.6 & 77.0 \\
\hline Foot disease & 37 & 1,040 & 7.2 & 16.8 & 18.0 \\
\hline Reproductive conditions & 37 & 2,022 & 14.0 & 32.9 & 27.0 \\
\hline Dry cow therapy & 31 & 2,018 & 13.9 & 39.3 & 38.1 \\
\hline Displaced abomasum & 28 & 482 & 3.3 & 10.6 & 11.3 \\
\hline Respiratory disease & 16 & 463 & 3.2 & 17.1 & 15.4 \\
\hline Fever & 15 & 205 & 1.4 & 8.1 & 10.0 \\
\hline Other & 37 & 1,200 & 8.3 & 19.4 & 22.4 \\
\hline Unknown & 20 & 941 & 6.5 & 27.7 & 28.0 \\
\hline Unclear & 2 & 5 & 0.0 & 1.5 & 1.1 \\
\hline
\end{tabular}

${ }^{1}$ Herds with at least 1 treatment for this condition in the TR.

\section{Association of ADD from TR and INV}

The Pearson product-moment correlation coefficient between overall ADD recorded using TR and INV was 0.80, indicating a strong positive correlation (Figure 2). When evaluating cubic, quadratic, and linear terms, a model allowing for 1 point of inflexion resulted in the best fit.

\section{DISCUSSION}

Quantification of AMU is essential to assess the relationship between AMU and AMR in humans and animals (Collignon and Voss, 2015). The association between the AMU in livestock and emergence of AMR in human bacterial isolates is under discussion (Seiffert et al., 2013). A restriction on AMU in animals is be- ing contemplated, as well as potential effects of such restrictions. Rigorous methodology is needed to accurately assess the overall quantity of antimicrobials used in livestock. Although it is critical that farmers and veterinarians promote prudent and judicious use of antimicrobials (Barkema et al., 2015), dairy herd-specific and time-sensitive methods of obtaining a measurement of AMU have not been implemented, precluding accurate assessments of the amount of antimicrobials used in a herd. Both methods used in this study were easy to implement, and challenges associated with them were related to interpretation of results and appropriate evaluation of the limitation of those techniques.

Estimated AMU was clearly higher for all evaluated antimicrobials using the INV than using the TR. An inherent bias to this type of study is the absence of blinding from the parts involved in recording of treat-

Table 4. Overall and geometric mean number of antimicrobial defined-daily doses (ADD) recorded using inventory of empty drug containers (INV) and treatment records (TR)

\begin{tabular}{|c|c|c|c|c|}
\hline \multirow[b]{2}{*}{ Antimicrobials } & \multirow[b]{2}{*}{ Herds $^{1}$} & \multicolumn{2}{|c|}{ Geometric mean } & \multirow[b]{2}{*}{$P$-value } \\
\hline & & INV & $\mathrm{TR}$ & \\
\hline Penicillins & 50 & 121.7 & 51.8 & $<0.001$ \\
\hline Penicillin combination & 49 & 69.8 & 31.0 & $<0.001$ \\
\hline Third-generation cephalosporins & 48 & 117.0 & 29.5 & $<0.001$ \\
\hline First-generation cephalosporins & 45 & 31.3 & 17.6 & 0.002 \\
\hline Trimethoprim-sulfonamide & 40 & 38.3 & 15.3 & $<0.001$ \\
\hline Tetracyclines & 39 & 21.6 & 8.1 & 0.004 \\
\hline Lincosamides & 32 & 25.0 & 7.4 & $<0.001$ \\
\hline Phenicols & 26 & 17.1 & 3.3 & 0.001 \\
\hline Macrolides & 25 & 15.8 & 3.2 & 0.001 \\
\hline Fluoroquinolones & 3 & - & - & - \\
\hline Other combinations & 2 & - & - & - \\
\hline Aminoglycosides & 1 & - & - & - \\
\hline Overall $^{3}$ & 51 & 536.6 & 196.0 & $<0.001$ \\
\hline
\end{tabular}

${ }^{1}$ Herds with at least 1 treatment with this antimicrobial in the TR or INV.

${ }^{2} P$-value for the comparison between TR and INV of the estimated means for various antimicrobials.

${ }^{3}$ Overall model fitted with maximum likelihood, Bayesian information criterion $=302$, deviance $=275$. 


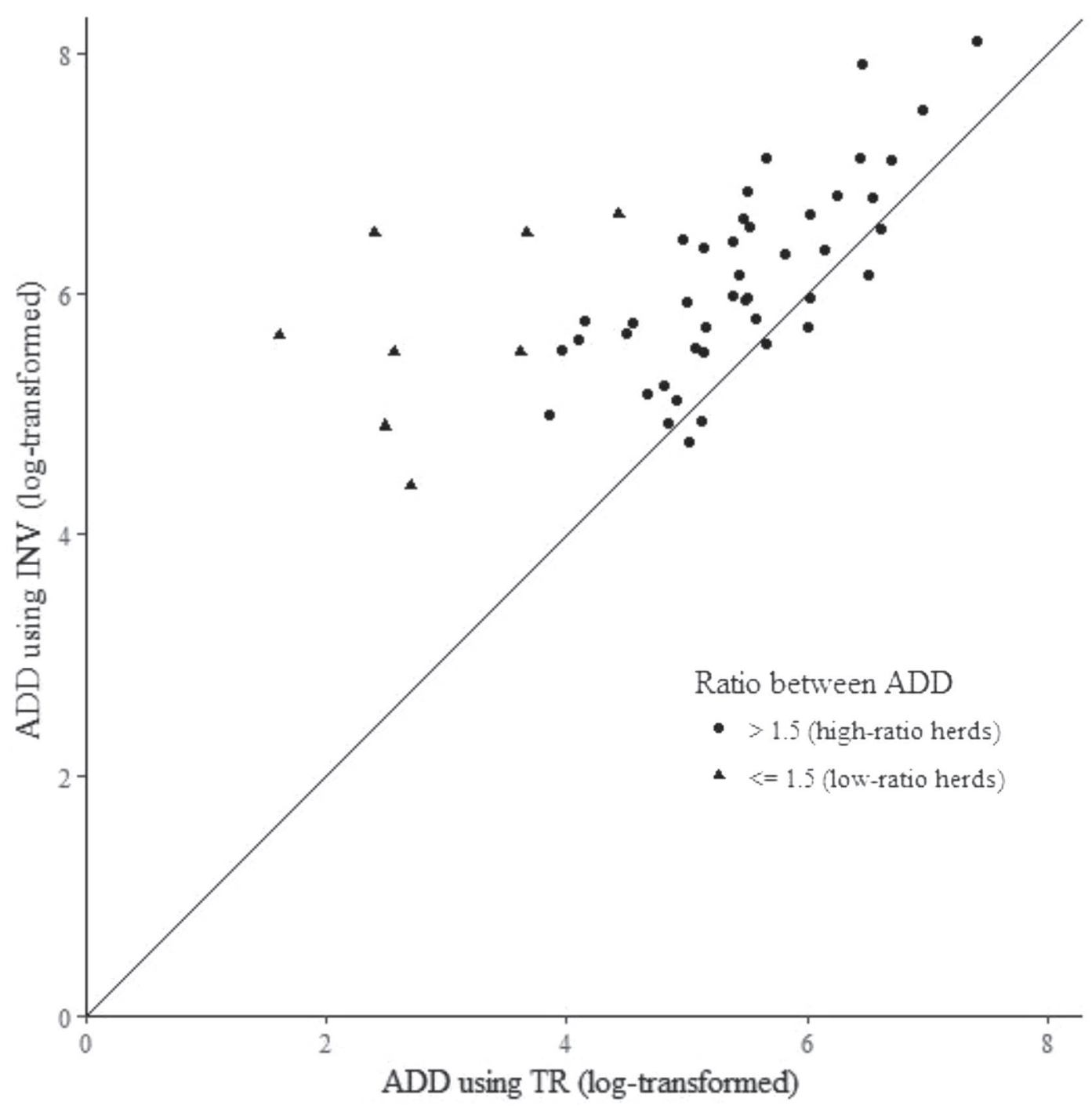

Figure 2. Scatterplot of total antimicrobial defined-daily doses (ADD) estimated using the treatment records (TR) and inventory of empty drug containers (INV) for 51 Canadian dairy herds.

ments. In that regard, it is very likely that receptacles were reminders to record treatments using the TR. Consequently, the number of ADD using the TR could, in theory, be even lower in a hypothetical absence of the garbage cans, as is generally the case in nonstudied herds. This could affect the extent to which this method can be used to estimate herd-level AMU. Noteworthy, TR are commonly used in trials and other designs as a measure of exposure to antimicrobials (Tragesser et al., 2006; Pol and Ruegg, 2007), often without considering limitations inherent to this data collection method. Hence, results of the present study could serve as a reference for researchers designing future studies that involves AMU as either exposure or outcome in dairy herds.
The additional AMU captured using the INV can be considered the true AMU or an overestimation of the AMU. In the first case, we assume that some treatments were not recorded using the TR. The TR can be assumed to have a high specificity to record an antimicrobial therapy. There are very limited circumstances in which treatments that did not happen were recorded. Conversely, TR may underestimate the number of true treatments, likely due to recall bias (Rhoda and Pantoja, 2012) or simply not keeping records up to date. Because there is ongoing pressure for proper recording of AMU in animals, TR are the easiest option for dairy producers to keep track of the herd AMU. However, the deficit observed in the present study could be detrimental if TR were used to estimate the total AMU in dairy 
herds. The INV, therefore, provided a better estimation of the number of antimicrobial therapies used in a herd than the evaluation of the TR. However, the number of treatments calculated using the INV could also be an overestimation of the actual number of treatments rendered in a farm. Potential reasons for this include off-label therapies, overdosing of antimicrobials, and discarding not completely empty containers. More than $90 \%$ of records in our TR were missing information about volume or dose used. If treatments were done using a higher dose than recommended, the INV would result in an overestimation of the total number of therapies. That a high percentage of dairy farmers rely on their personal experience for dosages used (Zwald et al., 2004) increases the chances of misclassification by the INV. Still, it is unrealistic to assume that $100 \%$ of the volume or quantity inside a bottle was properly used. Hence, it is very likely that the INV slightly overestimates the actual number of treatments. In contrast, an underestimation could be present as well, if not all empty containers were placed inside the garbage can or if the antimicrobial was frequently used to treat young animals. Regardless, caution is required when using either of these methods as a measure of exposure to antimicrobials.

When discussing AMU in dairy herds, methods used to capture this information should be acknowledged. Overall, ADD is a standardized measure of AMU that, although it does not reflect the actual use in field conditions, provides a quantity that reflects the total amount of drugs that are used, no matter where, how, and when. The influence of large herds in the overall ADD is high, as their overall number of empty containers/number of records on TR are on average higher than for smaller herds. However, a logarithmic transformation (as done in the present study) reduces the leverage of extreme values. The ADDR per year represents ADD divided by the years. This takes into consideration that herds were not necessarily observed for the same time period and AMU is a herd-specific variable. In a hypothetical scenario, if herds that used only macrolides were observed for a shorter interval than herds that used only penicillins, the overall ADD would be biased if we wanted to make comparisons between these 2 antimicrobials. The ADUR extends this concept to the number of animals per herd, and therefore should be a more reliable estimate for comparing AMU in different herds (Saini et al., 2012). In the present study, ADDR and ADUR for a specific antimicrobial were estimated conditional on the use of the same, which provided a measurement of the relative importance of a drug within a herd that uses the product. In that instance, drugs used intensively by a few herds would have a high value of $\mathrm{ADDR} / \mathrm{ADUR}$ and a low value of total $\mathrm{ADD}$.
Taken together, the 3 variables provided a complete description of the use and relative importance of the various antimicrobials in the studied herds. In the present study, the choice of the parameter to be compared when contrasting the INV and the TR appeared to be irrelevant, as the overall difference between methods was clear for all 3 parameters.

This study provided a description of antimicrobial treatments recorded in Canadian dairy herds, including reasons for therapies. Beta-lactams and their combinations were the most commonly used drugs for mastitis treatments worldwide (Brunton et al., 2012). Mastitis is reported to be the most frequent reason for antimicrobial treatment in dairy cows (Mitchell et al., 1998), followed by pneumonia and enteritis. As expected, penicillins, their combinations, and cephalosporins were the most commonly used antimicrobials at the animal level (Saini et al., 2012). However, frequent uses of trimethoprim and sulfonamide combinations and tetracyclines were surprising because those drugs are commonly used in dairy herds for foot-rot therapy and respiratory diseases (Sawant et al., 2005) and not for diseases of the udder, as was the case with the trimethoprim and sulfonamide combination.

A quadratic association existed between the $\log \mathrm{ADD}$ recorded using the 2 methods. In general, herds that recorded more treatments also placed more empty containers in the garbage cans. Therefore, we inferred that both methods were measuring the same underlying factor, namely, the relative AMU in the dairy herd, but with great variability. Some herds had an almost 1:1 association between the 2 methods, whereas other herds had up to 100 times higher ADD estimated using the INV compared with the TR. Herds that had a high ratio between the number of ADD recorded using both methods had a higher 2-yr average BTSCC versus the low-ratio herds. Bulk tank SCC is commonly used as an indicator of raw milk quality (Pantoja et al., 2009), serving as a parameter to be evaluated when assessing effects of herd-level management practices. The association between BTSCC and quality of the TR is very likely indirect. In a Dutch study, a high percentage of low-BTSCC farmers were classified as "clean and accurate," whereas a high percentage of high-BTSCC farmers were classified as "quick and dirty" (Barkema et al., 1999). In particular, record keeping and collection of clinical mastitis samples was on average less in high-BTSCC herds. Discarding empty drug containers in garbage cans is easier than keeping TR up to date. The latter method will therefore be better adhered to by farmers of low-BTSCC herds than their colleagues with high-BTSCC herds. It is also reassuring that, unlike the TR, the INV method showed counts of ADD that were not in an exceptional range of values for 
these herds, probably closer to the "true" number of ADD that was actually used. Nevertheless, the presence of low-ratio herds was undoubtedly responsible for the quadratic association between the 2 methods, introducing a great degree of variability with regard to estimating AMU on the farm. In the presence of such variability, it remains a challenge to predict the total AMU of one of the methods using the second; however, some insights might be gained when looking at mean or expected values.

Using the estimated parameters, it was possible to use the ADD recorded using the TR to obtain the expected ADD using the INV for the observed herds, especially by taking into account the supposed underestimation in the ADD obtained using TR.

In our study, 51 of 89 herds were able to initiate and implement a method of recording total AMU on the farm. The majority of herds that did not enter the study (28 of 38) used an alternative to the GHEF to record a health event. Six of the remaining 10 herds can be considered small compared with the average herd size in Canada. All 6 did not record a single treatment using the TR, whereas they all had used treatments using the INV. Unfortunately, reasons for the absence of records were not available, which would contribute to the understanding of potential limitations of this method. Nevertheless, it is noteworthy that all 89 herds in the present study were able to initiate and implement a method of recording total AMU on the farm.

In comparison to methods used in surveillance programs on AMU in livestock that assumed a constant use in all herds (i.e., sales data), the INV provided a herd-level specific quantity of AMU that correlated with AMU recorded at animal level using TR, and was established as a reliable tool for measuring total AMU used in a dairy herd. This study demonstrated that producers were willing to initiate protocols for recording AMU, and country-wide efforts should be encouraged to obtain such valuable data, as currently initiated in Canada within the proAction initiative (https://www .dairyfarmers.ca/proaction).

\section{CONCLUSIONS}

The estimated mean number of antimicrobial doses was 2.2 times higher using the INV compared with using the TR, with a clear association between the 2 methods. Both methods were easy to implement, and could provide valuable information assessing risk factors for AMR. Mastitis, reproductive conditions, and dry cow therapy were the most frequent reasons for implementing antimicrobial therapy in studied herds, whereas penicillins and its combinations, cephalosporins, trimethoprim and sulfonamide combinations, and tetracyclines were the most commonly used antimicrobials.

\section{ACKNOWLEDGMENTS}

This work was partially funded through the NSERC Industrial Research Chair in Infectious Diseases of Dairy Cattle. This project was also part of the Canadian Bovine Mastitis and Milk Quality Research Network program, funded by Dairy Farmers of Canada and Agriculture and Agri-Food Canada through the Dairy Research Cluster 2 Program. Three of the authors (DBN, SAN, SN) were supported by an NSERC-CREATE in Milk Quality scholarship. One author (DBN) was also supported by the Alberta Innovates-Technology Futures (Alberta, Canada). The authors thank all dairy producers, animal health technicians, and Canadian Bovine Mastitis Research Network (CBMRN) regional coordinators (Trevor De Vries, University of Guelph; Jean-Philippe Roy and Luc Des Côteaux, University of Montreal; Kristen Reyher, University of Prince Edward Island; and Herman Barkema, University of Calgary) that participated in data collection. The CBMRN data collections were financed by the Natural Sciences and Engineering Research Council of Canada (Ottawa, ON, Canada), Alberta Milk (Edmonton, AB, Canada), Dairy Farmers of New Brunswick (Sussex, New Brunswick, Canada), Dairy Farmers of Nova Scotia (Lower Truro, NS, Canada), Dairy Farmers of Ontario (Mississauga, ON, Canada) and Dairy Farmers of Prince Edward Island (Charlottetown, PE, Canada), Novalait Inc. (Québec City, QC, Canada), Dairy Farmers of Canada (Ottawa, ON, Canada), Canadian Dairy Network (Guelph, ON, Canada), Agriculture and Agri-Food Canada (Ottawa, ON, Canada), Public Health Agency of Canada (Ottawa, ON, Canada), Technology PEI Inc. (Charlottetown, PE, Canada), Université de Montréal (Montréal, QC, Canada), and University of Prince Edward Island (Charlottetown, PE, Canada), through the CBMQRN (Saint-Hyacinthe, QC, Canada).

\section{REFERENCES}

Barkema, H. W., J. D. Van der Ploeg, Y. H. Schukken, T. J. Lam, G. Benedictus, and A. Brand. 1999. Management style and its association with bulk milk somatic cell count and incidence rate of clinical mastitis. J. Dairy Sci. 82:1655-1663.

Barkema, H. W., M. A. von Keyserlingk, J. P. Kastelic, T. J. Lam, C. Luby, J. P. Roy, S. J. LeBlanc, G. P. Keefe, and D. F. Kelton. 2015. Invited review: Changes in the dairy industry affecting dairy cattle health and welfare. J. Dairy Sci. 98:7426-7445.

Bates, D., M. Mächler, B. Bolker, and S. Walker. 2015. Fitting linear mixed-effects models using lme4. J. Stat. Softw. 67:48.

Bondt, N., V. F. Jensen, L. F. Puister-Jansen, and I. M. van Geijlswijk. 2013. Comparing antimicrobial exposure based on sales data. Prev. Vet. Med. 108:10-20. 
Brunton, L. A., D. Duncan, N. G. Coldham, L. C. Snow, and J. R. Jones. 2012. A survey of antimicrobial usage on dairy farms and waste milk feeding practices in England and Wales. Vet. Rec. 171:296.

Bryan, M., and S. Y. Hea. 2017. A survey of antimicrobial use in dairy cows from farms in four regions of New Zealand. N. Z. Vet. J. 65:93-98.

Canadian Dairy Information Centre. 2017. Number of Farms, Dairy Cows and Heifers. Accessed Jul. 19, 2017. http://www.dairyinfo.gc .ca/index_e.php?s1=dff-fcil\&s2=farm-ferme\&s3=nb.

Carson, C. A., R. Reid-Smith, R. J. Irwin, W. S. Martin, and S. A. McEwen. 2008. Antimicrobial use on 24 beef farms in Ontario. Can. J. Vet. Res. 72:109-118.

Collignon, P., and A. Voss. 2015. China, what antibiotics and what volumes are used in food production animals? Antimicrob. Resist. Infect. Control 4:16.

Compendium of Veterinary Products. 2015. Compendium of Veterinary Products (CVP), 14th ed. North American Compendiums, Hensall, ON, Canada.

Crawley, M. J. 2013. The R Book. 2nd ed. Wiley, Chichester, UK.

Eagar, H., G. Swan, and M. van Vuuren. 2012. A survey of antimicrobial usage in animals in South Africa with specific reference to food animals. J. S. Afr. Vet. Assoc. 83:16.

Gonzalez, S. M., A. Steiner, B. Gassner, and G. Regula. 2010. Antimicrobial use in Swiss dairy farms: Quantification and evaluation of data quality. Prev. Vet. Med. 95:50-63.

Jensen, V. F., E. Jacobsen, and F. Bager. 2004. Veterinary antimicrobial-usage statistics based on standardized measures of dosage. Prev. Vet. Med. 64:201-215.

Landers, T. F., B. Cohen, T. E. Wittum, and E. L. Larson. 2012. A review of antibiotic use in food animals: perspective, policy, and potential. Public Health Rep. 127:4-22.

Mitchell, J. M., M. W. Griffiths, S. A. McEwen, W. B. McNab, and A. J. Yee. 1998. Antimicrobial drug residues in milk and meat: Causes, concerns, prevalence, regulations, tests, and test performance. J. Food Prot. 61:742-756.

Olde Riekerink, R. G., H. W. Barkema, D. T. Scholl, D. E. Poole, and D. F. Kelton. 2010. Management practices associated with the bulk-milk prevalence of Staphylococcus aureus in Canadian dairy farms. Prev. Vet. Med. 97:20-28.

Pantoja, J. C., D. J. Reinemann, and P. L. Ruegg. 2009. Associations among milk quality indicators in raw bulk milk. J. Dairy Sci. 92:4978-4987.

Pol, M., and P. L. Ruegg. 2007. Treatment practices and quantification of antimicrobial drug usage in conventional and organic dairy farms in Wisconsin. J. Dairy Sci. 90:249-261.

Queenan, K., B. Hasler, and J. Rushton. 2016. A One Health approach to antimicrobial resistance surveillance: is there a business case for it? Int. J. Antimicrob. Agents 48:422-427.

Reyher, K. K., S. Dufour, H. W. Barkema, L. Des Coteaux, T. J. Devries, I. R. Dohoo, G. P. Keefe, J. P. Roy, and D. T. Scholl 2011. The National Cohort of Dairy Farms-A data collection platform for mastitis research in Canada. J. Dairy Sci. 94:1616-1626.

Rhoda, D. A., and J. C. Pantoja. 2012. Using mastitis records and somatic cell count data. Vet. Clin. North Am. Food Anim. Pract. $28: 347-361$.

Saini, V., J. T. McClure, D. Leger, S. Dufour, A. G. Sheldon, D. T. Scholl, and H. W. Barkema. 2012. Antimicrobial use on Canadian dairy farms. J. Dairy Sci. 95:1209-1221.

Sawant, A. A., L. M. Sordillo, and B. M. Jayarao. 2005. A survey on antibiotic usage in dairy herds in Pennsylvania. J. Dairy Sci. 88:2991-2999.

Seiffert, S. N., M. Hilty, V. Perreten, and A. Endimiani. 2013. Extended-spectrum cephalosporin-resistant Gram-negative organisms in livestock: An emerging problem for human health? Drug Resist. Updat. 16:22-45.

Tragesser, L. A., T. E. Wittum, J. A. Funk, P. L. Winokur, and P. J. Rajala-Schultz. 2006. Association between ceftiofur use and isolation of Escherichia coli with reduced susceptibility to ceftriaxone from fecal samples of dairy cows. Am. J. Vet. Res. 67:1696-1700.

Zwald, A. G., P. L. Ruegg, J. B. Kaneene, L. D. Warnick, S. J. Wells, C. Fossler, and L. W. Halbert. 2004. Management practices and reported antimicrobial usage on conventional and organic dairy farms. J. Dairy Sci. 87:191-201. 